\title{
THE RELATIONSHIP BETWEEN SEXUAL OUTCROSSING AND HETEROKARYON INCOMPATIBILITY IN ASPERGILLUS NIDULANS
}

\author{
A. C. BUTCHER \\ Department of Genetics, University of Birmingham
}

Received 21.xii.67

\section{INTRODUCTION}

IT has often been assumed that in the homothallic Ascomycete Aspergillus nidulans, heterokaryon formation, the bringing together of unlike nuclei into the same cytoplasm, is a prerequisite of both parasexual and sexual recombination (Pontecorvo et al., 1953; Grindle, 1963b; Jinks and Grindle, 1963; Caten and Jinks, 1966). Grindle has shown, however (1963a and $b$ ) that, in this fungus, heterokaryosis, as measured by heterokaryotic conidial head formation, is limited to pairs of wild isolates which have very similar surface morphologies, and that wild isolates can be classified into different heterokaryon compatibility groups (h-c groups) upon the basis of their abilities to form heterokaryons with each other. Jinks and Grindle (1963) have demonstrated, by means of the heterokaryon transfer test, that heterokaryon compatibility is not controlled by non-chromosomal genes. It appears from this evidence that genetic exchange, via both the parasexual and sexual cycles, is limited in its occurrence to individuals with similar genotypes, at least in part, and that groups of compatible isolates, rather than the species as a whole are the units of evolution (Caten and Jinks, 1966).

Jinks, Caten, Simchen and Croft (1966) have shown, however, that the assumption that heterokaryon incompatibility is by necessity a barrier to sexual outcrossing is false by demonstrating the formation of hybrid perithecia between pairs of heterokaryon incompatibile isolates. It could well be, therefore, that wild isolates of $A$. nidulans are capable of outcrossing on a much wider scale than was previously supposed.

Heterokaryosis has been demonstrated in many homothallic ascomycetes (see Caten and Jinks, 1966) and systems of heterokaryon compatibility, similar to that found in Aspergillus nidulans, demonstrated in other Aspergillus species (Caten, 1965; Jones, 1966) and in Neurospora crassa (Garnjobst and Wilson, 1956). The evaluation of the respective importance of sexual and parasexual crossing in genetic exchange in $A$. nidulans is, therefore, of great consequence for the study of the genetics of natural populations of fungi.

\section{Materials and methods}

All the isolates used were either wild types from the Birmingham collection which were obtained from sites scattered throughout England and Wales or U.V. induced conidial colour mutant derivatives of these isolates. The latter bore either yellow $(y)$ or white $(w)$ conidia instead of the wild-type green conidia.

Two crossing programmes were carried out. In the first, 17 wild-type 
isolates and their mutant derivative strains were used, each wild-type isolate representing a different h-c group. In the second programme 3 wild-type isolates, plus their respective mutant strains, belonging to each of 4 randomly selected h-c groups were used. Thus information could be obtained about both intra- and inter-h-c group relationships.

The crossing procedure for each programme was identical and as follows. In any single cross one parent was a wild type isolate and the other a mutant derivative strain, thus enabling perithecia of hybrid origin to be instantly recognised. In each crossing programme crosses were made both between wild-type isolates and their own mutant derivative strains ("selfing" crosses), e.g. $1 \times 1 y$, and between wild-type isolates and mutant derivative strains of other wild-type isolates, e.g. $1 \times 4 y$ and $1 y \times 4$. The first programme therefore consisted of 289 individual crosses and the second of 144 crosses.

Individual matings were set up by inoculating Petri dishes, containing Czapeck minimal agar, with $0.15 \mathrm{ml}$. of heavy conidial suspensions of each parent, so that at least $10^{6}$ conidia were put onto each plate. The suspensions were thoroughly mixed and spread over the agar surface, and the inoculated plates incubated at $25^{\circ} \mathrm{C}$. for at least a fortnight before perithecia were sampled. Because of the size of the two crossing programmes, individual crosses were set up and incubated in successive weekly batches, to which they were allocated at random. The batch size was determined by the number of crosses that could be sampled in a week, which was approximately 20 with a sample size of 20 perithecia per cross. Within each batch the crosses were sampled in a random order. At least eight perithecia were sampled and scored in each cross.

Individual plates were sampled by picking off mature perithecia formed in areas of the mating culture where both conidial colours, could be seen to be well intermingled. These perithecia were then rolled across a sterile agar surface, in order to remove the surrounding Hülle cells and any mycellium or conidial heads, and squashed on a clean area of the same surface so that a pool of ascospores was released. The perithecium wall was discarded. A sample of ascospores from each perithecium was transferred, on the bulbous end of a glass needle, to a drop of "Tween 80 " on a separate sterile agar surface. The drop of liquid was spread over the agar surface and the plate incubated at $25^{\circ} \mathrm{C}$. for 4 days. At the end of this period the conidial colour of the colonies resulting from ascospore germination was readily visible to the naked eye.

This technique did not allow any information about ascospore viability to be gained since it was impossible to know how many ascospores were transferred in each sample. It was, however, preferred to the more usual sampling methods involving suspension and dilution to standard concentrations because it allowed many more perithecia to be sampled in the same period of time. Nevertheless, any serious upset in viability would be immediately apparent and, with practice, it was possible to obtain samples of a fairly consistent size.

The individual perithecia sampled were scored as either selfed or hybrid in origin. A selfed perithecium was one whose ascospores gave rise to only all wild-type or all mutant colonies, whereas a hybrid perithecium was one whose ascospores gave rise to wild-type and mutant colonies in a ratio not significantly different from $1: 1$. Furthermore, where any other visible characters dependent upon a single gene difference were present, these were 
checked for $1: 1$ segregation in the hybrid perithecia. Cases where wild-type and mutant colonies occurred in ratios significantly different from $1: 1$ were classified as selfs contaminated with the other parent and scored as the appropriate self. This occurred in no more than 2-3 per cent. of all perithecia sampled and could well have been caused by faulty cleaning of the perithecia before bursting or possibly by the occurrence of twin perithecia inside single perithecial walls.

\section{Results}

Although hybrid perithecia were present in some crosses, but not in others, there was no evidence, in either crossing programme, of any systematic grouping of these crosses which could be accounted for by a system of sexual incompatibility. However, when the number of hybrid perithecia found in each cross was expressed as a proportion of the total number of perithecia sampled from that cross, it was apparent that marked variation existed between individual crosses, which appeared to be due, in part, to differences between the individual parent isolates.

Table 1

Analysis of variance of the first crossing programme, involving 17 unrelated wild isolates of A. nidulans, each belonging to a different $h-c$ group

$\begin{array}{crcc}\text { Item } & \text { d.f. } & \text { M.S. } \\ a \text { Additive effects } & 16 & 524 \cdot 4538 * * * \\ b \text { Non-additive effects } & 136 & 124 \cdot 5476 \\ c+d \text { Error } & 136 & 124 \cdot 7409 \\ * * * \text { Significant at } \mathrm{P}=0 \cdot 001 .\end{array}$

Owing to the proportional nature of the data, it was necessary to apply the angular transformation $p=\sin ^{2} \varnothing$ (where $p$ is the observed proportion of hybrid perithecia in each case), before any statistical analysis could be performed. This transformation is insufficient for very low values of $p$. Therefore 0.002 was added to each value before transformation.

The statistical analysis used was Hayman's analysis of variance of a diallel cross (Hayman, 1954). The designs of the two crossing programmes can be regarded, in many respects, as $17 \times 17$ and $12 \times 12$ sets of diallel crosses respectively. In the absence of recognisable male and female parents, the analyses were carried out with respect to wild-type and mutant parents. Whereas in a more conventional diallel analysis, material effects (items $c$ and $d$ in the analysis) could have been measured, these were replaced, in this particular case, by effects attributable to the presence of conidal colour markers $(y$ or $w)$ in the wild genotypes. As the mutant strains used were chosen to differ from their respective wild-type isolates by only a single gene controlling conidial colouration, which previous authors (Grindle, $1963 a$ and $b$; Jinks $e t a l ., 1966$ ) have shown to have no effect upon any characters in which they were interested, these effects were attributed to error variation. Therefore in the analyses of variance (tables 1, 2 and 4), items $c$ and $d$ have been pooled (item $c+d$ ) to provide an estimate of the error.

Item $b$ would normally have been a test for dominance but, as the exact analogy of this effect in a haploid system is difficult to describe, it can be presumed that item $b$ tests for the non-additive effects of the parental isolates on rate of outcrossing. Item $a$ tests whether or not there is an additive effect of each isolate on rate of outcrossing. 
In the analysis of the first crossing programme (table 1), item $a$ was highly significant when tested against item $c+d$, whereas item $b$ was nonsignificant. Similar results were obtained from the analysis of the second crossing programme (table 2 (i)).

TABLE 2

Analysis of variance of the second crossing programme involving 12 wild isolates; 3 selected from each of 4 h-c groups

(i) Overall analysis

$\begin{array}{rcc}\text { Item } & \text { d.f. } & \text { M.S. } \\ a & 11 & 457 \cdot 7108 * * * \\ b & 66 & 83 \cdot 3725 \\ c+d & 66 & 96 \cdot 2658\end{array}$

(ii) Complete analysis

Item d.f. M.S.

Between $h-c$ groups

$\begin{array}{rcc}a & 3 & 1138 \cdot 3625^{*} \\ b & 6 & 217 \cdot 8147 \\ c+d & 6 & 211 \cdot 4930\end{array}$

Within h-c groups

$\begin{array}{rrc}a & 8 & 202 \cdot 3413^{*} \\ b & 60 & 69 \cdot 9282 \\ c+d & 60 & 84 \cdot 7632\end{array}$

*** Significant at $\mathrm{P}=0.001$. * Significant at $\mathrm{P}=0.05$.

There was, therefore, a statistically additive effect of each parental isolate on rate of sexual outcrossing. It follows from this that the proportion of hybrid perithecia in any one "selfing" cross should be related to the average proportion of hybrid perithecia in all the crosses involving that isolate. In table 3 the selfing, rate taken as the angular transformation of the proportion of hybrid perithecia in the " selfing" cross, and the outcrossing rate, taken as the mean of the angular transformations of the proportions of hybrid perithecia in all crosses involving the parents of the self, are tabulated for each parental isolate in both crossing programmes. There was a highly significant correlation between these two characters in both programmes.

It can be concluded from these results that each wild isolate of $A$. nidulans has a characteristic crossing potential and that the rate of outcrossing between any two parents (including "selfing" crosses) can be predicted from the sum of their respective crossing potentials.

There is now evidence (Jinks et al., 1966) that isolates belonging to the same h-c group have similar genotypes. It is to be expected, therefore, that all isolates within any single $\mathrm{h}$-c group will have similar crossing potentials. The design of the second crossing programme allows this hypothesis to be tested in that it is possible to sum results over h-c groups to produce a $4 \times 4$ table. This enables separate $a, b$ and $c+d$ items to be calculated for between h-c group variation and for within h-c group variation. The analysis of variance of these items (table 2 (ii)) shows that significant additive variation occurs both between and within h-c groups, the former being significant when tested against the latter. 
At first sight these results appear to disprove the hypothesis that, within any single h-c group all isolates have similar crossing potentials. It is possible, however, to demonstrate that significant additive variation occurs within only one of the four h-c groups (table 4), and it is therefore, reasonable to conclude that variation between wild isolates belonging to the same $\mathrm{h}-\mathrm{c}$ group is the exception rather than the rule.

TABLE 3

Selfing and outcrossing rates of the wild isolates used in each crossing programme

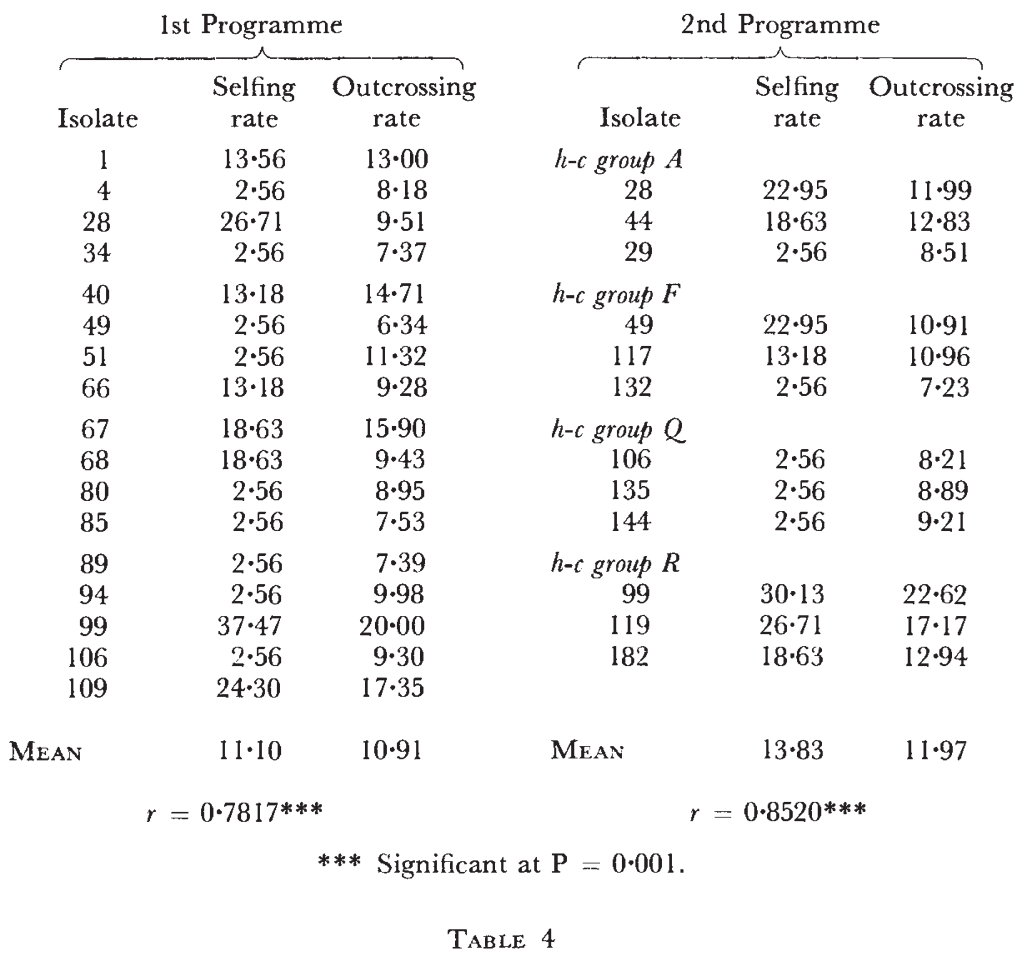

Breakdown of the within $h-c$ group items a and $\mathrm{c}+\mathrm{d}$ from table 2 (i) into separate items for variation within $h-c$ group, and the analysis of variance of these items

$\begin{array}{ccc} & a \text { M.S. } & c+d \text { M.S. } \\ \text { h-c group } & (\text { d.f. }=2) & (\text { d.f. }=15) \\ \text { A } & 127.883 & 123.425 \\ \text { F } & 110.199 & 55.124 \\ \text { Q } & 6.288 & 61.589 \\ \text { R } & 575.599 * & 112.827 \\ & \text { * Significant at } \mathrm{P}=0.05 .\end{array}$

A statistically additive system controlling rate of outcrossing can, presumably act in one of three ways. These are (i) through a differential suppression of free outcrossing, (ii) through a differential promotion of free outcrossing, or (iii) through a combination of these two.

It was possible to decide between these three alternatives by testing whether the three types of perithecia within any one cross, namely selfed 
wild-type, selfed mutant and hybrid occurred with random frequencies. This was carried out by applying the Hardy-Weinberg equation and thus calculating, from the observed frequencies of the three types of perithecia, frequencies to be expected if outcrossing were at random. Whether or not the observed frequencies differed significantly from the expected was then

TABLE 5

Classification of the intividual crosses in both programmes according to the randomness of crossing. The number of crosses in each category is expressed as a percentage of the total number of crosses in the programme. For full explanation of the classification see text

\begin{tabular}{lcc}
\multicolumn{1}{c}{ Category } & $\begin{array}{c}\text { lst Programme } \\
\% \text { total crosses }\end{array}$ & $\begin{array}{c}\text { 2nd Programme } \\
\% \text { total crosses }\end{array}$ \\
1. Greater than random & 0.35 & 0.00 \\
2. Less than random & 53.97 & $65 \cdot 28$ \\
3. Random & $22 \cdot 13$ & $13 \cdot 19$ \\
4. One class of perithecia & 21.45 & 16.67 \\
5. No perithecia & 0.35 & 4.86 \\
6. Insufficient data & 1.73 & 0.00
\end{tabular}

tested by the chi-squared test $($ d.f. $=1)$. The results obtained are summarised in table 5 . On the basis of this test the crosses can be classified into three main categories:

(1) Crosses in which there were significantly more hybrid perithecia than would be expected if crossing were at random.

(2) Crosses in which there were significantly less hybrid perithecia.

(3) Crosses in which the number of hybrid perithecia did not differ significantly from that expected if crossing were at random.

In addition, the following three minor categories could be recognised and classified separately:

(4) Crosses where the perithecia were either all selfed wild-type or all selfed mutant.

(5) Crosses which yielded no perithecia.

(6) Crosses where insufficient data was recorded for reliable classification to be made.

The majority of crosses were of category (2), while most of the remainder were of categories (3) and (4). There was only one cross of category (1). The majority of crosses of category (3) involved at least one parent with a high crossing potential. Thus it appears that the additive control acts through a suppression rather than a promotion of free outcrossing.

These findings are not consistent with the phenomenon of "relative heterothallism" reported by Hemmons, Pontecorvo and Bufton (Pontecorvo et al., 1953) who found many crosses yielding between 50 per cent. and 100 per cent. hybrid perithecia. It must be remembered however that these authors worked with auxotrophic mutant strains derived from one original wild isolate, whereas the present work is concerned with many different prototrophic wild isolates. It is, therefore, not surprising that their findings cannot be extrapolated to the behaviour of natural populations. 


\section{Discussion}

(i) The nature of the control of sexual outcrossing

It now seems reasonably clear that the failure of pairs of wild isolates of $A$. nidulans to form heterokaryotic conidial heads is a result of heterokaryon disadvantage (Caten and Jinks, 1966) rather than true incompatibility per se. This point of view was favoured by Jinks et al. (1966), who discussed the relevant evidence, both from their own work and from that of others. They did, in fact, show that progeny from inter-h-c group crosses were less vigorous, as determined by growth rate studies, than their parents, whereas progeny from intra-h-c group crosses were, on average, as vigorous as their parents. Thus there was a strong suggestion that the genomes of isolates of differing h-c groups were mutually unbalanced and hence it would be expected that heterokaryons between such isolates would be at a disadvantage to the homokaryotic balanced parents.

Assuming that inter-h-c group anastomoses do occur, as they must for hybrid perithecium formation, but that the heterokaryons so formed fail to establish themselves, it can be inferred that the formation of hybird perithecia must occur very soon after anastomosis.

It could be that the initial heterokaryon can persist long enough for the initial dikaryon of the ascogenous hypha to form. Indeed, the formation of the dikaryon may actually be favoured by the fact that the heterokaryon is at a disadvantage. There is evidence from basidiomycetes (Simchen and Jinks, 1964), where dikaryons can be readily studied, that they can persist and flourish in the presence of parents which are far more vigorous. This possibility allows for sexual crossing in the absence of heterokaryotic conidial head formation.

It is also possible that, on the anastomosis of two heterokaryon incompatible homokaryons, a heterokaryotic dikaryon, rather than a heterokaryotic hypha, is immediately formed and gives rise to a hybrid perithecium. This would imply, however, that the production of hybrid perithecia in a cross between two heterokaryon incompatible isolates occurs at a different stage to the production of selfed perithecia or perithecia of hybrid origin in a cross between two heterokaryon compatible isolates.

A third possibility is that anastomoses leading to sexual outcrossing, and anastomoses leading to vegetative hyphal fusion are of two distinct types. This postulation has the attraction that it allows heterokaryon disadvantage and an additive regulation of outcrossing rate to occur independently. Under such a system the crossing potential of any isolate would be some function of its ability to undergo special sexual anastomoses. There is, however, no reported evidence of the existence of such special anastomoses in $A$. nidulans.

In enumerating these possibilities, no consideration has been given to the nature of the additive control mechanism which has already been shown to act through a suppression of free outcrossing. Two possible levels at which such a suppression mechanism might act are (i) in controlling the proportion of nuclei of each isolate capable of entering into a heterokaryotic dikaryon or, (ii) in controlling the rate of anastomoses between hyphae of different genotypes. However, since the additive control system is active in " selfing" crosses $($ e.g. $49 \times 49 y)$ a difference in genotype of only one gene, i.ê. a conidial colour marker, is significant. 
It is unlikely that any model involving only one factor, e.g. rate of anastomosis, can account for the additive effect and it is probable that a more involved causal system, possibly relying on the interaction of several factors, is to be expected. It should be remembered that the measurement of crossing rate is proportional in nature and is therefore affected, not only by variation in the absolute number of hybrid perithecia produced but also by variation in the numbers of selfed perithecia of each parent.

It is interesting to note, at this juncture, that Garnjobst and Wilson (1956) have reported a system of heterokaryon incompatibility, in Neurospora crassa, consistent with the genotypes of the strains used and independent of the dipolar sexual compatibility system. These workers also demonstrated that anastomoses between incompatible individuals did in fact occur but an " incompatibility reaction" occurred after plasmogamy leading to the decay of the heterokaryotic portion of the mycelium.

\section{(ii) The importance of sexual outcrossing in natural populations}

Now that it has been demonstrated that heterokaryon incompatibility is not a barrier to sexual outcrossing, as was previously supposed, it would seem that, in $A$. nidulans, sexual outcrossing has a potentially greater role to play in genetic exchange than either heterokaryosis or parasexuality. This is by no means proven and the probable effects of outcrossing in natural populations must be considered in the light of existing knowledge.

For example, Grindle (1963c, and unpublished) has shown that members of the same h-c group can be isolated from sites scattered throughout England and Wales while at any given site members of more than one h-c group can be isolated. This work revealed no geographical clines in h-c groupings.

Jinks et al. (1966) have shown that the progeny from a cross between heterokaryon incompatible parents are, as a rule, heterokaryon incompatible with each other and with either parent. Thus free outcrossing would tend to breakdown compatibility groups. On the other hand, progeny from different crosses could well have similar enough genotypes to be heterokaryon compatible and therefore, given that inter-h-c group outcrossing does occur in natural populations, the geographical distribution obtained is to be expected.

Alternatively, studies carried out by Jinks et al. (1966) have demonstrated that the progeny of inter-h-c group outcrosses exhibit a reduced vigour in growth rate. Since growth rate is presumably a fitness character, this finding can be viewed as evidence that the progeny of inter-h-c group crosses are at a selective disadvantage and would probably have difficulty in establishing themselves in competition with their parents. If this is so then successful outcrossing, i.e. the leaving behind of successful progeny, will be limited to the same extent as heterokaryosis and parasexuality.

The observed geographical distribution of h-c groups can also be explained by postulating that each h-c group, and, therefore, each distinct genotype, is adapted to a different micro-environment and hence effectively isolated. At the present time it is impossible to choose between these alternatives although the comparative vigour of parents, and progeny of sexual crosses is currently being investigated further. Some circumstantial evidence can be gained, however, from consideration of the number of isolates classified into each recognised h-c group in the Birmingham collection. It can be 
postulated that, if successful sexual outcrossing occurs in the natural environment, a genotype with a high crossing potential is more likely to be broken down by inter-h-c group outcrossing than a genotype with a low crossing potential. This should be reflected in the number of members in individual $\mathrm{h}$-c groups. This, however, is not the case and therefore, some indication that successful inter-h-c group outcrossing does not occur in the natural environment.

Within h-c groups sexual outcrossing will have a varying importance in genetic exchange depending upon the group's characteristic crossing potential. The actual variation of crossing potential between h-c groups may well be a manifestation of an evolutionary trend towards the imperfect state exhibited by many Aspergilli, e.g. A. unguis (see Raper and Fennell, 1965).

\section{Summary}

1. Two separate crossing programmes were carried out. In the first of these 17 wild isolates of $A$. nidulans, each belonging to a separate heterokaryon compatibility group, and, in the second, 3 wild isolates from each of 4 different heterokaryon compatibility groups were crossed in all possible combinations. The proportion of perithecia of hybrid origin in each cross was recorded.

2. The results obtained clearly demonstrated that heterokaryon incompatibility was not a barrier to sexual outcrossing and was a result of heterokaryon disadvantage rather than the absence of anastomosis.

3. The proportion of hybrid perithecia recovered from any single cross was found to be predictable from the sum of crossing potentials characteristic of each parent. Every wild isolate tested was found to have a different crossing potential and these varied significantly between heterokaryon incompatible, but not between heterokaryon compatible isolates.

4. The additive control was shown to act through a suppression of free outcrossing.

5. Possible causes of the additive effect are discussed together with the probable significance of sexual outcrossing in genetic exchange within natural populations.

6. Comparisons are made between compatibility relationships in $A$. nidulans and Neurospora crassa.

Acknowledgments. - I wish to thank Professor J. L. Jinks and Dr J. H. Groft, for advice and constructive criticism. This work was supported by the Agricultural Research Council.

\section{REFERENCES}

CAten, C. E. 1965. Asexual systems of variation in fungi. Ph.D. thesis, University of Birmingham Library.

CATEN, C. E., AND Jinks, J. L. 1966. Heterokaryosis: its significance in wild homothallic Ascomycetes and fungi imperfecti. Trans. Brit. Mycol. Soc., 49, 81-93.

GARNJOBST, L., AND WILSON, J. F. 1956. Heterokaryosis and protoplasmic incompatibility in Neurospora crassa. Proc. Nat. Acad. Sci., 42, 613-618.

GRINDLE, M. 1963a. Heterokaryon compatibility of unrelated strains in the Aspergillus nidulans group. Heredity, 18, 191-204.

GRINDLE, M. 1963b. Heterokaryon compatibility of closely related wild isolates of Aspergillus nidulans. Heredity, 18, 397-405.

GRINDLE, M. 1963c. Nuclear and cytoplasmic variation in fungi. Ph.D. thesis, University of Birmingham Library.

hayman, в. I. 1954. The analysis of variance of diallel crosses. Biometrics, 10, 789-809. 
JINKS, J. L., CATEN, C. E., SIMCHEN, G., AND GROFT, J. C. 1966. Heterokaryon incompatibility and variation in wild populations of Aspergillus nidulans. Heredity, 21, 227-239.

JINKs, J. L., AND GRINDLE, M. 1963. The genetical basis of heterokaryon incompatibility in Aspergillus nidulans. Heredity, 21, 227-239.

JONEs, D. A. 1965. Heterokaryon incompatibility in the Aspergillus glaucus link group. Heredity, 20, 49-56.

PONTECORVO, G., ROPER, J. A., Hemmons, L. M., MAGDONALD, K. D., AND BUfTon, A. J. W. 1953. The genetics of Aspergillus nidulans. Adv. Genet., 5, 141-238.

RAPER, K. B., AND fenNell, D. I. 1965. The Genus Aspergillus. Williams and Wilkins Co., Baltimore.

Simchen, G., AND JiNKs, J. L. 1964. The determination of dikaryotic growth rate in the Basidiomycete Schizophyllum commune: a biometrical analysis. Heredity, 19, 629-649. 